\title{
A novel electrospun-aligned nanoyarn/three- dimensional porous nanofibrous hybrid scaffold for annulus fibrosus tissue engineering
}

This article was published in the following Dove Press journal: International Journal of Nanomedicine

\author{
Jun $\mathrm{Ma}^{1, *}$ \\ Yunfei $\mathrm{He}^{1,2, *}$ \\ Xilin Liu',* \\ Weiming Chen ${ }^{3}$ \\ An Wangl,4 \\ Chia-Ying $\operatorname{Lin}^{5,6}$ \\ Xiumei $\mathrm{Mo}^{3}$ \\ Xiaojian $\mathrm{Ye}^{1}$ \\ 'Department of Orthopaedics, \\ Changzheng Hospital, Second \\ Military Medical University, Shanghai, \\ ${ }^{2}$ Department of Spinal Surgery, Lanzhou \\ General Hospital of Lanzhou Military \\ Command Region, Lanzhou, ${ }^{3}$ College \\ of Chemistry, Chemical Engineering \\ and Biotechnology, Donghua University, \\ ${ }^{4}$ Department of Orthopaedics, Shanghai \\ Armed Police Force Hospital, Shanghai, \\ China; ${ }^{5}$ Structural Tissue Evaluation \\ and Engineering Laboratories, \\ Department of Biomedical Engineering, \\ ${ }^{6}$ Department of Orthopaedic Surgery, \\ University of Cincinnati, Cincinnati, \\ OH, USA \\ *These authors contributed equally \\ to this work
}

Correspondence: Xiaojian Ye Department of Orthopaedics, Changzheng Hospital, Second Military Medical University, 4I5 Fengyang Road,

Shanghai 200003, China

Tel +862181885624

Fax +86 2I 63720099

Email xjyespine@smmu.edu.cn

Xiumei Mo

College of Chemistry, Chemical Engineering and Biotechnology, Donghua University, 2999 Renmin Road North,

Shanghai 201620, China

Email xmm@dhu.edu.cn
Introduction: Herniation of the nucleus pulposus (NP) because of defects in the annulus fibrosus (AF) is a well-known cause of low back pain. Defects in the AF thus remain a surgical challenge, and efforts have been made to develop new techniques for closure and repair. In this study, we developed an electrospun aligned nanoyarn scaffold (AYS) and nanoyarn/threedimensional porous nanofibrous hybrid scaffold (HS) for AF tissue engineering.

Methods: The AYS was fabricated via conjugated electrospinning, while the aligned nanofibrous scaffold (AFS) was prepared by traditional electrospinning as a baseline scaffold. The HS was constructed by freeze-drying and cross-linking methods. Scanning electron microscopy and mechanical measurement were used to characterize the properties of these scaffolds. Bone marrow derived mesenchymal stem cells (BMSCs) were seeded on scaffolds, and cell proliferation was determined by CCK- 8 assay, while cell infiltration and differentiation were assessed by histological measurement and quantitative real-time polymerase chain reaction, respectively.

Results: Morphological measurements showed that AYS presented a relatively better three-dimensional structure with larger pore sizes, higher porosity, and better fibers' alignment compared to AFS. Mechanical testing demonstrated that the tensile property of AFS and AYS was qualitatively similar to the native AF tissue, albeit to a lesser extent. When BMSCs were seeded and cultured on these scaffolds, the number of cells cultured on HS and AYS was found to be significantly higher than that on AFS and culture plate after 7 days of culture $(P<0.05)$. In addition, cell infiltration was significantly higher in HS when compared with AFS and AYS $(P<0.05)$. A part of BMSCs ingressed into the inner part of AYS upon long-term in vitro culture. No significant difference was observed between AFS and AYS in terms of the median infiltration depth $(P>0.05)$. BMSCs seeded on AYS demonstrated an increased expression of COL1A1, while the expression levels of SOX-9, COL2A1, and Aggrecan were higher in HS compared to other scaffolds $(P<0.05)$.

Conclusion: These findings indicate that HS makes a proper scaffold for the AF tissue engineering as it replicates the axial compression and tensile property of AF, thereby providing a better platform for cell infiltration and cell-scaffold interaction.

Keywords: electrospinning, nanoyarn, three-dimensional scaffold, cell infiltration, annulus fibrosus, tissue engineering

\section{Introduction}

Herniation of the nucleus pulposus (NP) tissue through the annulus fibrosus (AF) defects is a well-known cause of low back pain, leg numbness, and weakness. ${ }^{1}$ Discectomy or microdiscectomy procedure is a well-known intervention for radicular pain resulting from NP herniation. ${ }^{2,3}$ Existing evidence shows that patients benefit from it when compared with nonoperative management. ${ }^{4,5}$ However, discectomy does not repair the remaining 
AF defect to restore the integrity of AF, and thus, re-herniation may occur at the rate ranging from 1 to $38 \%$ based on different kinds of annular defects. ${ }^{6}$ Carragee et $\mathrm{al}^{6}$ reported that larger AF defects postdiscectomy are risk factors for recurrent disk herniation and are associated with poor outcome. ${ }^{7}$

Currently available procedures for AF closure are designed to prevent recurrence, to restore mechanical integrity, and to promote repair of the injured tissue. Direct repair of annular incisions by suture techniques failed to restore intervertebral disk (IVD) intradiscal pressures and did not significantly alter the healing strength of the IVD in sheep model of AF defects. ${ }^{8} \mathrm{~A}$ recent multicenter randomized controlled clinical trial showed no effect of commercialized suturing techniques for AF closure (eg, Xclose C and Anulex Technologies Inc) on reduction in the rate of re-herniation. ${ }^{9}$ AF implants such as the Barricaid $^{\circledR}$ (Intrinsic Therapeutics, Woburn, MA, USA) prevent NP tissue re-herniation and should provide the surgeon with increased confidence in minimizing nucleus removal for pain relief, while preserving the disk height, and sustaining the disk biomechanics, retarding the degeneration process and associated poor clinical outcomes in long term. ${ }^{10-12}$ Some sutures, such as fibril hydrogels, have shown positive results in experimental studies to some extent. ${ }^{13}$ However, all these methods merely provide a physical barrier to prevent NP re-herniation and neither the device nor the sutures restore mechanical properties of the motion segment or promote AF tissue regeneration.

Tissue engineering provides an advanced strategy that applies an engineering approach for the regeneration of scaffolds that replaces and restores compromised tissues. To enable injured tissue to recover its normal function and promote tissue regeneration, tissue engineering $\mathrm{AF}$ scaffolds must mimic native tissue, both mechanically and physiologically. AF is a laminated angle-ply composite structure with layers oriented at $\sim \pm 30^{\circ}$ angles in the outer $\mathrm{AF}$ and $\sim \pm 45^{\circ}$ in the inner $\mathrm{AF}$, which imparts heterogeneity and highly anisotropic mechanical properties. ${ }^{14}$ Successful tissue engineering of AF scaffolds must match the material properties of AF as closely as possible.

Multiple techniques have been applied to fabricate scaffolds suitable for tissue engineering. Among these, electrospinning has been extensively used to create highly aligned arrays of polymeric nanofibers scaffold, scale, and architecture of which mimic the natural organization of many fiber-reinforced soft tissues, such as muscle, tendon, knee meniscus, and AF. ${ }^{15}$ These scaffolds can be manufactured to obtain essential mechanical behaviors of fiber-reinforced soft tissues such as nonlinearity, anisotropy, and finite elastic deformations. ${ }^{16}$ Attachment and proliferation of bovine and human AF cells and mesenchymal stem cells (MSCs) on electrospun scaffolds have been observed in different studies. ${ }^{17-19}$ Nerurkar et al used aligned nanofibrous scaffolds to engineer single lamellar AF constructs ${ }^{20}$ and to fabricate bi-lamellar tissues that replicate the angle-ply fiber organization to mimic native AF tissue. ${ }^{21}$ Another study constructed a disk-like angle-ply structure that replicated the multi-scale architecture of the IVD as a new approach for disk tissue engineering. ${ }^{22}$

However, owing to its dense fiber assembly, the cellular infiltration and matrix formation are limited to the AFS boundaries. Therefore, it is critical to improve the cell-scaffold interactions and cell infiltration for improved efficiency. Moreover, little attention has been paid to mechanical parameters, especially the axial compression property of the scaffold. In this study, a nanoyarn-porous nanofiber hybrid scaffold (HS) was fabricated by conjugated electrospinning and freeze-dry manner. The scaffolds were seeded with bone marrow-derived MSCs (BMSCs), and cell proliferation and scaffold infiltration were determined. The HS, which comprised a nanoyarn layer and a porous nanofiber layer, was most efficient in balancing the mechanical property and scaffold porosity and may provide a promising strategy to AF repair.

\section{Materials and methods Materials}

Poly(L-lactide-co-caprolactone) (P[LLA-CL]; poly[L-lactide] [PLLA]:PCL =75:25; molecular weight: 340,000 Da) was provided by GUNZE Co., Ltd. (Kyoto, Japan). Gelatin was purchased from MP Biomedicals (CA, USA). PLLA was provided by Medprin Regenerative Medical Technologies Co., Ltd (Guangzhou, China). Glutaraldehyde and tert-butanol were purchased from Sinopharm Chemical Reagent Co., Ltd (Shanghai, China). 1,1,1,3,3,3-Hexafluoro-2-propanol (HFIP) was purchased from Darui Co., Ltd. (Shanghai, China).

\section{Scaffold fabrications}

Figure 1 illustrates the process for scaffolds' fabrication. Briefly, $10 \mathrm{~mL}$ of gelatin solution (dissolved in $10 \mathrm{~mL}$ of HFIP at $12 \%, \mathrm{w} / \mathrm{v}$ ) and $3 \mathrm{~mL}$ of poly(lactic acid) (PLA) solution (dissolved in $10 \mathrm{~mL}$ of HFIP at $8 \%, \mathrm{w} / \mathrm{v}$ ) were blended together to form a homogeneous solution (PLA/ gelatin, weight ratio $=1: 5)$. The solution was charged with a voltage of $15 \mathrm{kV}$ and fed at a rate of $5.0 \mathrm{~mL} / \mathrm{h}$ with $9 \mathrm{G}$ metal needle using a syringe pump. The resultant nanofibers were deposited onto an aluminum foil at a height of $15 \mathrm{~cm}$ from the blunt tip. Three grams of gelatin/PLA nanofibers membranes were separately cut into small pieces $(1 \times 1 \mathrm{~cm})$ and dispersed in $100 \mathrm{~mL}$ tert-butanol by homogenizing the mixture for $15 \mathrm{~min}$ at $12,000 \mathrm{rpm}$ using an IKA T18 homogenizer (IKA-Werke GmbH \& Co., Staufen, Germary). 


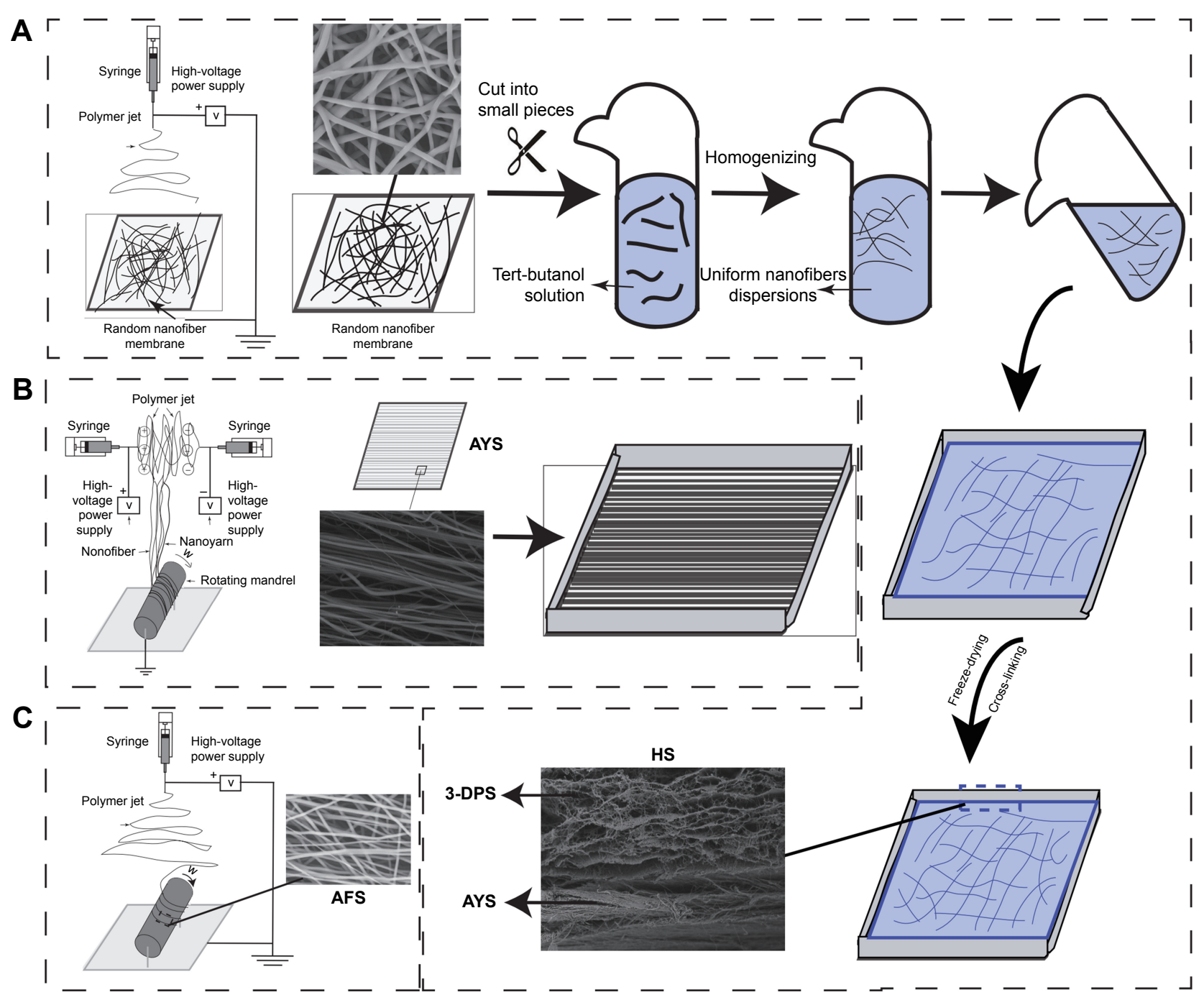

Figure I Schematic description of experimental procedure for different scaffolds.

Notes: (A) Schematic diagram of nanoyarn/three-dimensional porous nanofibrous HS construct process. 3-DPS were manufactured according to our previous work. Briefly, the uniform nanofibers' dispersions, which were homogenized from random nanofibers, were poured over the AYS with an aluminum mold. After freeze-drying and cross-linking, three-dimensional porous nanofibrous/nanoyarn HSs were obtained. (B) The gelatin/P(LLA-CL) solution (Wt 10\%) was used to produce AYS by conjugated electrospinning from two oppositely charged nozzles. (C) Gelatin/P(LLA-CL) AFS were prepared by traditional electrospinning process.

Abbreviations: AFS, aligned nanofiber scaffolds; AYS, aligned nanoyarn scaffolds; 3-DPS, three-dimensional porous nanofibrous scaffolds; HS, hybrid scaffold; P(LLA-CL), poly(L-lactide-co-caprolactone); PLLA, poly(L-lactide).

As has been shown previously that three-dimensional porous fiber scaffolds (3-DPS) with $3 \mathrm{~g}$ of gelatin/PLA have a comparable axial compression property-like AF (0.35 MPa), it was used in this study. ${ }^{23}$ Uniform nanofibers' dispersions were poured into a mold above the aligned nanoyarn scaffolds (AYS), frozen at $-80^{\circ} \mathrm{C}$ for $2 \mathrm{~h}$, and then freeze-dried for $24 \mathrm{~h}$ to obtain the unstable HS. ${ }^{24}$ Glutaraldehyde (5\%) was added to ethanol as a cross-linker. The unstable scaffolds were cross-linked by glutaraldehyde for $10 \mathrm{~min}$. To improve the biocompatibility of cross-linked scaffolds, they were treated with a $5 \%$ solution of $\mathrm{L}$-glutamic acid in $0.5 \mathrm{M}$ hydrochloric acid for $48 \mathrm{~h}$ and freeze-dried overnight. ${ }^{25}$

The gelatin/P(LLA-CL) blend (weight ratio: 20:80) was dissolved in HFIP at $10 \%(\mathrm{w} / \mathrm{v})$. After agitation for $24 \mathrm{~h}$, the gelatin/P(LLA-CL) solution was used for producing AYS by conjugated electrospinning. Polymer solutions were delivered to two spinnerets, which were horizontally opposite to each other and located above the grounded collector at a distance of $15 \mathrm{~cm}$. Two high electrical voltages of $12 \mathrm{kV}$ with opposite polarities were applied to the two spinnerets, and the solution jet impelling rate was set at $1.5 \mathrm{~mL} / \mathrm{h}$. The fibers from the two oppositely charged spinnerets carry opposite charges, which attract each other and strike together to form polymer composite nanoyarns. The yarns and part of unpacked fibers were continuously collected by the rotating mandrel at a rotation rate of $140 \mathrm{rpm}$. Nanoyarns of $\sim 250 \mu \mathrm{m}$ thickness were electrospun to match the natural lamellar thickness of the AF. ${ }^{26}$ The $\mathrm{AFS}^{27}$ were 
constructed according to our previous protocols with some modification. ${ }^{23}$ All these scaffolds were stored in a vacuum oven for 7 days, then immersed in $75 \%$ ethanol for $4 \mathrm{~h}$, and washed with phosphate-buffered saline (PBS) for three times at $30 \mathrm{~min}$ each before use.

\section{Morphological characterization of the scaffolds}

The surface morphology of those scaffolds was examined by scanning electron microscope (SEM; JSM-5600, JEOL, Tokyo, Japan). Before scanning, platinum was coated on the surface of the scaffolds twice for $10 \mathrm{~s}$ each. The fiber diameter, nanoyarn bundle diameter, and angle distribution (relative to the vertical axis) of the specimens $(n=3)$ were determined using Image J 1.47 (National Institute of Health, Washington D.C, USA). The angle distribution and mean diameter were determined for more than 100 randomly selected nanofibers and nanoyarns. The orientation of the fiber (or nanoyarn) was determined by measuring the angle between the fiber (or nanoyarn) and the direction of mandrel rotation for AFS and AYS. The mean pore size was determined for $>100$ pores. A pore was identified as a void space surrounded by fibers (or nanoyarns) on all sides and nearly the same depth. The porosity of the scaffolds was calculated using a previously described method $(n=4) .{ }^{28}$

\section{Measurement of mechanical properties of scaffolds}

After measuring the cross-sectional areas using a caliper, scaffold samples were cut into $50 \times 10 \mathrm{~mm}$ rectangular samples, which were clamped with serrated grips and loaded onto an optical microscope and digital image correlation testing device. Tensile tests were performed using a constant crosshead speed of $10 \mathrm{~mm} / \mathrm{min}$ until failure. Strain was determined as extension normalized to the gauge length and stress was computed as the load normalized to the initial cross-sectional area. Young's modulus was computed as a slope of the stress-strain plot, determined by regression of the linear portion of the curve. The tensile strength and elongation at the break were determined from the maximum stress and maximum strain before material failure $(n=5)$.

\section{BMSCs' isolation and culture}

Primary BMSCs from Sprague Dawley rats were harvested and cultured using a previously described protocol. ${ }^{29}$ All the protocols were approved by the Institutional Animal Care and Use Committee of Second Military Medical University
(Shanghai, China). All animal handling procedures were performed according to the Guide for the Care and Use of Laboratory Animals of Second Military Medical University. Following euthanasia, the ends of the femora were removed and marrow of the midshaft flushed out with 1-mL syringe. Marrow was suspended in a standard medium (SM) with Dulbecco's Modified Eagle's Medium (DMEM) - high glucose (Thermo Fisher Scientific, Waltham, MA, USA), $10 \%$ fetal bovine serum (FBS) (Thermo Fisher Scientific) and $1 \%$ penicillin/streptomycin (Thermo Fisher Scientific) and plated in $25 \mathrm{~cm}^{2}$ flasks (Corning Incorporated, Corning, NY, USA) at a density of $1 \times 10^{6}$ cells $/ \mathrm{mL}$. Non-adherent cells were removed after $24 \mathrm{~h}$, and the medium was replaced every 2-3 days. Experiments were carried after the second passage. Upon reaching confluency, BMSCs were removed by $0.25 \%$ trypsin in $1 \mathrm{mM}$ ethylenediaminetetraacetic acid tetrasodium (Thermo Fisher Scientific) and plated on the pretreated scaffolds.

For differentiation studies, BMSCs were seeded at a density of $5 \times 10^{5}$ cells per scaffold in a chondrogenic medium (CM). CM consisted of DMEM - high glucose, supplemented with 10\% FBS, 4 mM L-glutamine (Sigma-Aldrich Co., St Louis, MO, USA), 0.1 $\mu \mathrm{M}$ dexamethasone, $0.17 \mathrm{mM}$ ascorbic acid-2-phosphate, $0.1 \mu \mathrm{M}$ ITS + Premix Culture Supplement (Corning Incorporated), $1 \mathrm{mM}$ sodium pyruvate, $0.35 \mathrm{mM}$ proline, $1 \%$ penicillin/streptomycin (Thermo Fisher Scientific), and $10 \mathrm{ng} / \mathrm{mL}$ transforming growth factor- $\beta 3$ (TGF-ß3) (R\&D Systems, Inc., Minneapolis, MN, USA). The medium was changed every 2 days and evaluated for chondrogenic markers at day 14.

\section{BMSCs' proliferation on scaffolds}

Cell viability was determined by measuring cell metabolic activity using the cell counting kit-8 (CCK-8; Dojindo, Kumamoto, Japan) according to the manufacturer's instructions. To quantify cell proliferation, a proliferation assay was performed on every scaffold. A sample with a surface area of $\sim 32 \mathrm{~mm}^{2}$ was cut off from each scaffold and placed in a 96-well plate containing $100 \mu \mathrm{L}$ of culture medium. Viable cell number was determined by absorbance at $450 \mathrm{~nm}$ in a microplate reader from BioTek (Winooski, VT, USA).

\section{Morphology of BMSCs on scaffolds}

The cell-cultured scaffolds were processed for SEM studies after 7 days of seeding. The scaffolds were rinsed twice with PBS and fixed in $2.5 \%$ glutaraldehyde for $3 \mathrm{~h}$. Thereafter, the scaffolds were dehydrated with increasing concentrations of ethanol $(60,70,80,90$, and $100 \%)$ for 15 min each. 
Finally, the scaffolds were sputter coated with gold and then observed under SEM.

\section{Histology}

The scaffolds were preimmersed in culture medium overnight before cell seeding. After cell seeding with a density of $3 \times 10^{6}$ cells/scaffold, the cell-scaffold constructs were incubated for $4 \mathrm{~h}$ to allow the cells to completely adhere to the scaffolds. Then, the constructs were placed in a six-well plate and incubated in the normal medium as described earlier. After incubation for 14 days, the samples were fixed in $4 \%$ paraformaldehyde and $5 \mu \mathrm{m}$ cross-sections were obtained after dehydration, clarification, infiltration, and paraffin embedding. The sections were processed for hematoxylin and eosin (HE) staining (Sigma-Aldrich Co.), and images were obtained using a microscope (DM4000 B; Leica Microsystems, Wetzlar, Germany).

\section{Cell infiltration measurements}

For cellular infiltration assay, the constructs were collected at each time point and flash frozen in liquid $\mathrm{N}_{2}$. Samples were embedded in optimal cutting temperature (OCT) compound (Tissue-Tek; Sakura Finetechnical Co. Ltd., Tokyo, Japan), cut into $10 \mu \mathrm{m}$ sections using a Leica cryo-microtome, and mounted onto poly(L-lysine) precoated microscope slides. The sections were then fixed in $4 \%$ polyformaldehyde for $30 \mathrm{~min}$ at room temperature. Visualization of cells in the scaffold was done by staining the fixed sections with the DNA-binding stain 4',6-diamidino-2-phenylindole (DAPI). Quantification of the depth of cellular infiltration was performed with a modified method previously described by Pham et $a l^{30}$ with some modifications. For this purpose, a set of columns of $10 \mu \mathrm{m}$ widths and $20 \mu \mathrm{m}$ apart from each other were overlaid. The deepest distance (relative to the mesh surface) was measured and recorded in each column. The samples per group per time point and at least three sections per sample were analyzed to obtain the quantitative infiltration depth data. Point-to-point distance measurements were performed using Image J 1.47.

\section{Quantitative real-time polymerase chain reaction (PCR)}

Total RNA was extracted from cell-loaded scaffold using TRIzol reagent (Thermo Fisher Scientific) following the manufacturer's instructions. The cDNA was synthesized with moloney murine leukemia virus reverse transcription reagents (Takara, Kusatsu, Shiga, Japan). Housekeeping gene GAPDH was used as a normalization control. Real-time PCR was conducted using the SYBR Premix qPCR kit (Takara). Data were analyzed by
Table I Primer sets used for PCR measurements

\begin{tabular}{llll}
\hline Name & Primer & Sequence & $\begin{array}{l}\text { Size } \\
\text { (bp) }\end{array}$ \\
\hline Rat GAPDH & Forward & 5'-ACAGCAACAGGGTGGTGGAC-3' & 253 \\
& Reverse & 5'-TTTGAGGGTGCAGCGAACTT-3' & \\
Rat COLIAI & Forward & 5'-TGACTGGAAGAGCGGAGAGT-3' & 202 \\
& Reverse & 5'-GAATCCATCGGTCATGCTCT-3 & \\
Rat Aggrecan & Forward & 5'-TGGACTTGTCTCAGGTTTC-3' & 295 \\
& Reverse & 5'-AGTTGGGGCAGTTATGGAT-3 & \\
Rat SOX-9 & Forward & 5'-CAGTCCCAGAGAACGCACAT-3' & 232 \\
& Reverse & 5'-AGGTGAAGGTGGAGTAGAGC-3 & \\
Rat COL2AI & Forward & 5'-TCCCGCAAGAATCCCGCTCG-3' & 164 \\
& Reverse & 5'-GCTGGGTTGGGGTAGACGCA-3 & \\
\hline
\end{tabular}

the $\Delta \Delta \mathrm{CT}$ method for relative gene expression. The primers are listed in Table 1. Each sample was tested in triplicate.

\section{Statistical analysis}

All experiments were conducted at least three times, and normally distributed data are presented as mean \pm standard deviation (SD) or presented as median and interquartile range. Single factor one-way analysis of variance (ANOVA) was used to determine statistically significant difference $(P<0.05)$ between groups in normally distributed data, while nonparametric Kruskal-Wallis test was used for non-normally distributed data. All statistical analyses were performed using SPSS Statistics Version 19.0 (IBM Corporation, Armonk, NY, USA).

\section{Results}

\section{Electrospun scaffold morphology}

The surface morphology of AFS, AYS, and HS and cross-sectional structures of HS are shown in Figure 2. Images of the AFS (Figure 2A) revealed that the scaffolds were composed of aligned nanofibers, while those in AYS (Figure 2B) were composed of aligned nanoyarns (marked with red ellipses and arrows) and nanofibers, presenting a relatively three-dimensional structure. The random arrangement PLLA/gelatin nanofiber membranes, which were used to form the 3-DPS, are shown in Figure 2C. HS (Figure 2D) was formed by AYS and 3-DPS layer by layer. AYS (Figure 2B) and 3-DPS (Figure 2E) exhibited a three-dimensional porous structure, especially the latter one, while AFS (Figure 2A) consisted of densely packed nanofibers. Figure 2E and $\mathrm{F}$ shows the fiber morphology of 3-DPS in HS before and after cross-linking with 5\% glutaraldehyde, showing no significant change in nanofibers' morphology.

Nanofibers in AFS exhibited a relatively uniform fiber alignment and large part of nanofibers formed angles ranging from 0 to $30^{\circ}$ (Figure $3 \mathrm{~A}$ ). Most of the nanoyarns and nanofibers in 

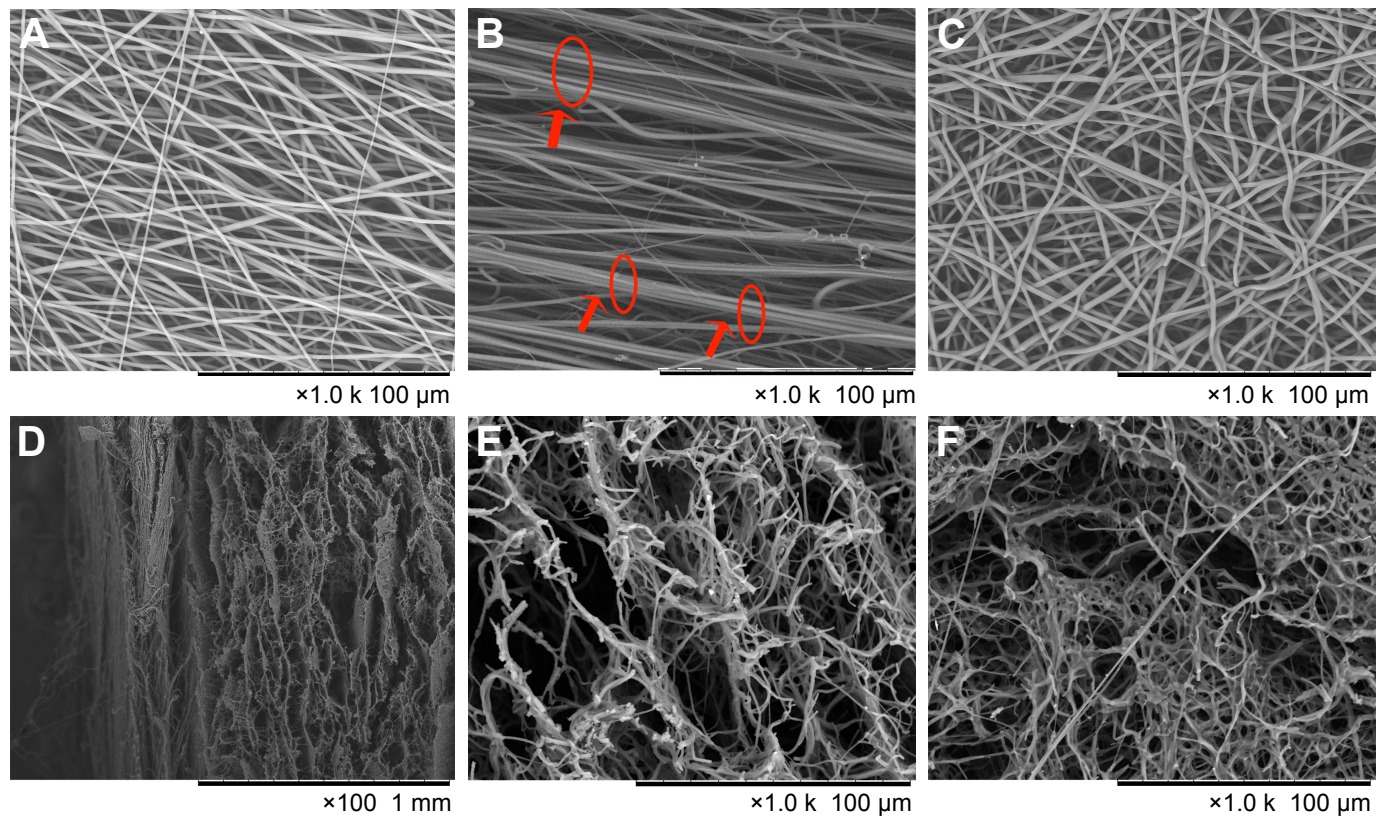

Figure 2 SEM images of electrospinning scaffolds

Notes: (A) Aligned gelatin/P(LLA-CL) nanofibers scaffold; (B) aligned gelatin/P(LLA-CL) nanoyarn fibers scaffold; and (C) random PLLA/gelatin fibers. (D) Cross-section image of hybrid scaffold. Three-dimensional porous nanofibrous scaffold, before cross-linked (E) and after cross-linked (F). Red arrows and ellipses in (B) indicate the formation of nanoyarn fibers.

Abbreviations: P(LLA-CL), poly(L-lactide-co-caprolactone); PLLA, poly(L-lactide); SEM, scanning electron microscope.

AYS showed better fiber alignment, forming angles ranging from 0 to $20^{\circ}$ (Figure 3B). As shown in Figure 3C, the median pore size of AFS (median [quartile 1, quartile 3]) was 59.69 (32.88, 90.33) $\mu \mathrm{m}^{2}$, which was significantly smaller than AYS with a median pore size of $240.61(140.62,450.40) \mu \mathrm{m}^{2}(P<0.05)$ and 3-DPS with a median pore size of $314.70(177.04,446.57) \mu \mathrm{m}^{2}$ $(P<0.05)$. There was no significant difference between AYS and 3-DPS in terms of pore size $(P>0.05)$. Some large pores, which were $>2,000 \mu \mathrm{m}^{2}$, were observed in the 3-DPS group. In addition, 3-DPS exhibited a significantly higher porosity of $83.3 \% \pm 1.17 \%$ compared to AFS $(71.7 \% \pm 1.09 \%, P<0.05)$ and AYS (79.7\% $\pm 2.01 \%, P<0.05$ ) (Figure 3D).

\section{Mechanical properties of electrospun scaffolds}

Typical stress-strain curves of AFS and AYS are shown in Figure 4A and B, respectively. A 6.5-fold increase in tensile strength in the parallel direction (13.46 $\pm 0.54 \mathrm{MPa})$ compared to the perpendicular direction $(2.02 \pm 0.23 \mathrm{MPa})$ was observed in AFS $(P<0.05)$. A more robust increase $(13$-fold) was observed in AYS $(10.39 \pm 0.46 \mathrm{MPa}$ in parallel direction vs $0.75 \pm 0.02 \mathrm{MPa}$ in perpendicular direction) $(P<0.05)$ (Figure 4C). Similarly, Young's modulus in direction parallel to the rotation of the mandrel was 75 and 35 times greater than that in perpendicular direction $(159.46 \pm 13.05$ vs $2.06 \pm 0.93$ and $111.19 \pm 5.57$ vs $3.17 \pm 0.50 \mathrm{MPa}$, respectively) $(P<0.05)$ (Figure 4D). However, the elongation at break values of AFS and AYS in the parallel direction was significantly smaller than that in the perpendicular direction $(60.83 \% \pm 3.12 \%$ vs $201.01 \% \pm 8.43 \%, P<0.05$, and $112.48 \% \pm 2.13 \%$ vs $197.78 \% \pm 9.92 \%, P<0.05$, respectively) (Figure 4E). Both AFS and AYS exhibited notable anisotropic mechanical properties as significant differences in the mechanical properties in the parallel and perpendicular directions of both scaffolds. Both Young's modulus and tensile strength in the parallel direction were significantly larger in AFS than in AYS $(P<0.05)$.

\section{Cell proliferation on scaffolds}

BMSCs $\left(7.5 \times 10^{3}\right.$ cells) were seeded and cultured on tissue culture plate (TCP), AFS, AYS, and HS and imaged using a SEM at day 7. The optical density (OD) values shown in Figure 5 indicated that there was a slight increase in the number of cells in the AFS $(0.940 \pm 0.016, P<0.05)$ and AYS $(0.942 \pm 0.021, P<0.05)$ groups after 1 day of culture compared to TCP $(0.866 \pm 0.041)$, while it decreased in the HS $(0.701 \pm 0.015, P<0.05)$ group. The cell proliferation rate on AYS and HS was significantly higher than that on TCP and AFS. No significant difference was observed between TCP and AFS at days 3 and 7.

\section{Cell morphology imaged by SEM}

SEM was used to examine the influence of culture environment on cell morphology and cell-scaffold interaction. 
A

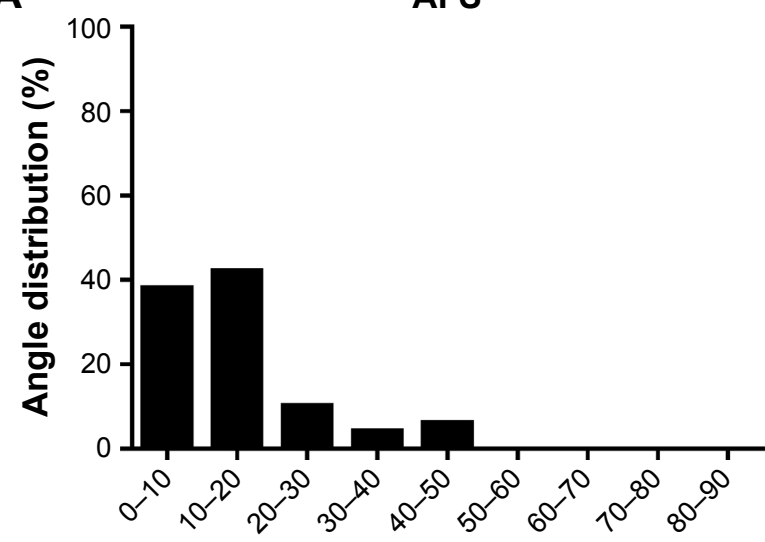

Angle $\left({ }^{\circ}\right)$

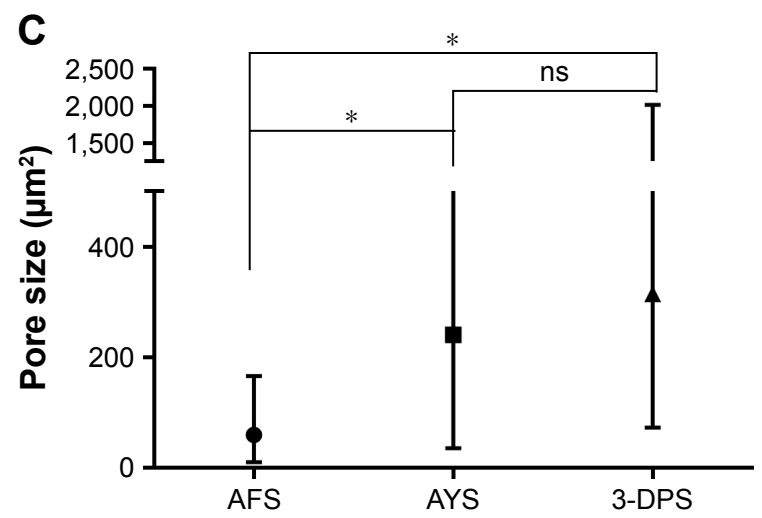

B

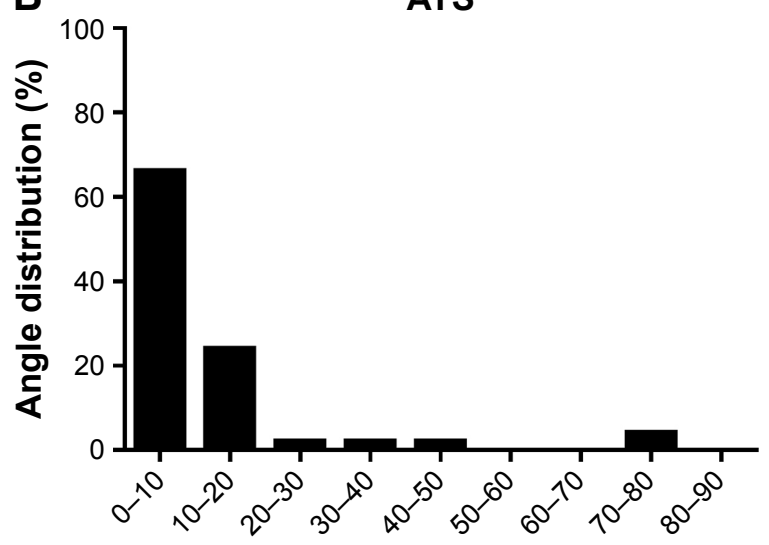

Angle $\left({ }^{\circ}\right)$

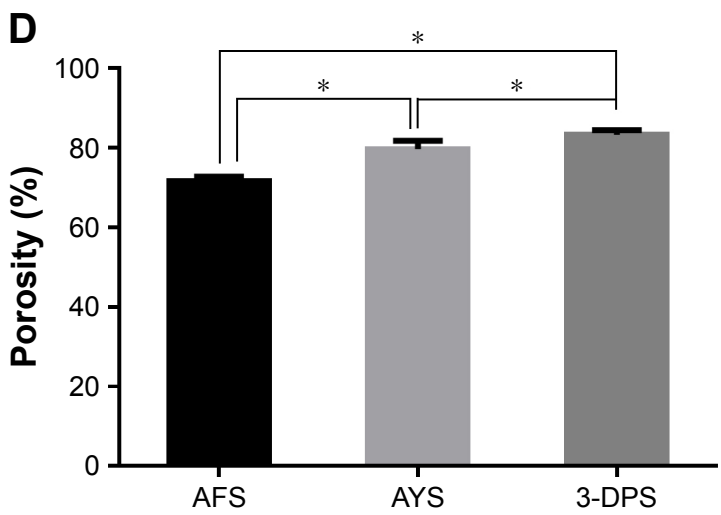

Figure 3 Representative structure characteristics of scaffolds.

Notes: Angular distribution of the nanofibers in the AFS (A) and the nanoyarns in the AYS (B). (C) Pore size of AFS, AYS, and 3-DPS, measured by Image J. The data are expressed as median + interquartile range. (D) Porosity of AFS, AYS, and HS. The data are expressed as mean \pm SD. $* P<0.05$, a statistically significant difference between the two groups; ns, $P>0.05$, no significant difference between the two groups.

Abbreviations: AFS, aligned nanofiber scaffolds; AYS, aligned nanoyarn scaffolds; 3-DPS, three-dimensional porous nanofibrous scaffolds; HS, hybrid scaffold; SD, standard deviation.

Figure 6 shows the morphology of BMSCs in individual scaffolds. SEM images of cell cultures observed on day 7 showed normal morphology of mesenchymal cells in AFS, AYS, and HS. Cells in AFS showed normal extensions and typical elongated morphology (Figure 6A). Cells were oriented along the direction of the fibers and clustered around the nanoyarn fibers in a longitudinal fashion in AYS (Figure 6B). In contrast, BMSCs in HS aggregated to form small colonies (Figure 6C). The increased cell density in HS and AYS as compared to cells in AFS was consistent with the result from the cell proliferation experiment.

\section{Histological analyses}

The cell penetration and gross distribution of BMSCs seeded scaffolds were determined by HE staining (Figure 7). While HS showed large cell penetration and growth on both the surface and porous areas (Figure 7C, D, G, H, K, and L), AYS showed less cell distribution compared to HS (Figure 7B, $\mathrm{F}$, and $\mathrm{J}$ ), and a part of cells infiltrated into the center of the scaffold. Limited cell infiltration in the periphery of the scaffold was observed in AFS (Figure 7A, E, and I).

\section{Cell infiltration}

To verify the presence and distribution of BMSCs in the scaffold, DAPI staining was performed (Figures 8 and 9). After a 3-day culture, the median and interquartile range of infiltration depth of BMSCs in AFS (Figures 8A and 9B), AYS (Figures 8B and 9B), and HS (Figures 8C and 9B) were found to be $9.88(6.53,10.85), 12.64(11.65,19.50)$, and $236.43(185.87,267.44) \mu \mathrm{m}$, respectively $(P<0.05)$. By day 7, an increase in the number of infiltrated cells was observed in HS (Figures 8F and 9B), while only a limited increase was observed in AFS (Figures 8D and 9B) $(P<0.05)$. After a 14-day culture, the BMSCs had migrated to a depth of $414.34(380.95,457.05) \mu \mathrm{m}$ in HS (Figures 8I and 9B), $55.60(17.06,112.10) \mu \mathrm{m}$ in AYS (Figures 8H and 9B), and $34.65(26.62,36.76) \mu \mathrm{m}$ in AYS (Figures $8 \mathrm{H}$ and 9B) $(P<0.05)$. Notably, a small part of BMSCs had ingrown 

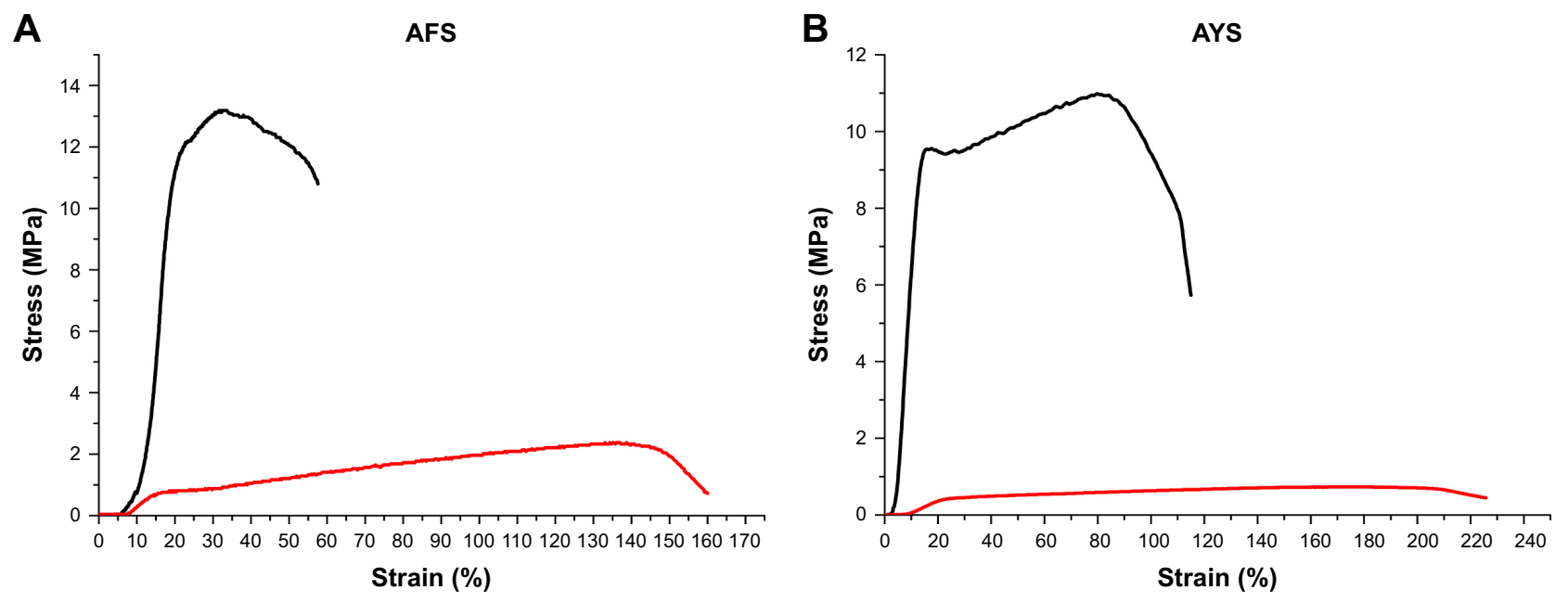

C

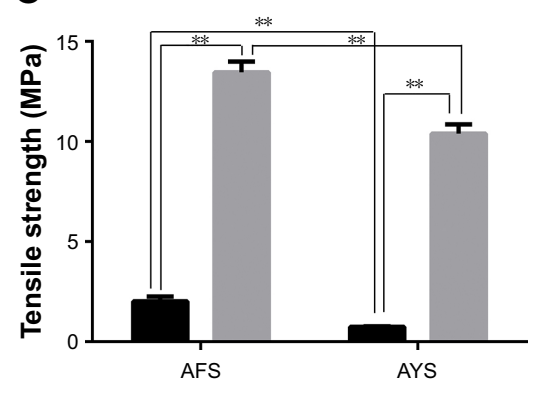

D

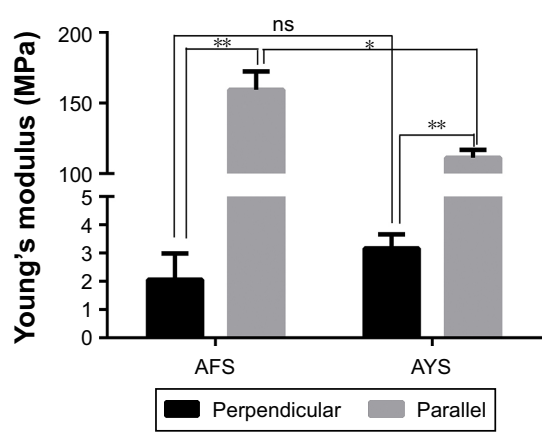

E

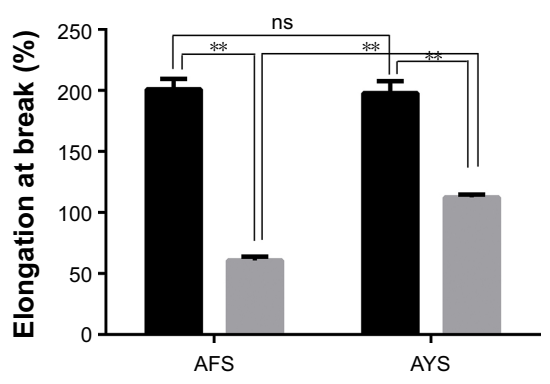

Figure 4 Mechanical properties of scaffolds.

Notes: Representative tensile stress-strain curve of AFS (A) and AYS (B); (C) tensile strength at break; (D) Young's modulus; and (E) elongation rate at break of P(LLA-CL)/ gelatin AFS and AYS. The data are expressed as mean \pm SD. $* P<0.05$, $* * P<0.01$, a statistically significant difference between groups; ns, $P>0.05$, no significant difference between the two groups $(n=3)$.

Abbreviations: AFS, aligned nanofiber scaffolds; AYS, aligned nanoyarn scaffolds; P(LLA-CL), poly(I-lactide-co-caprolactone); SD, standard deviation.

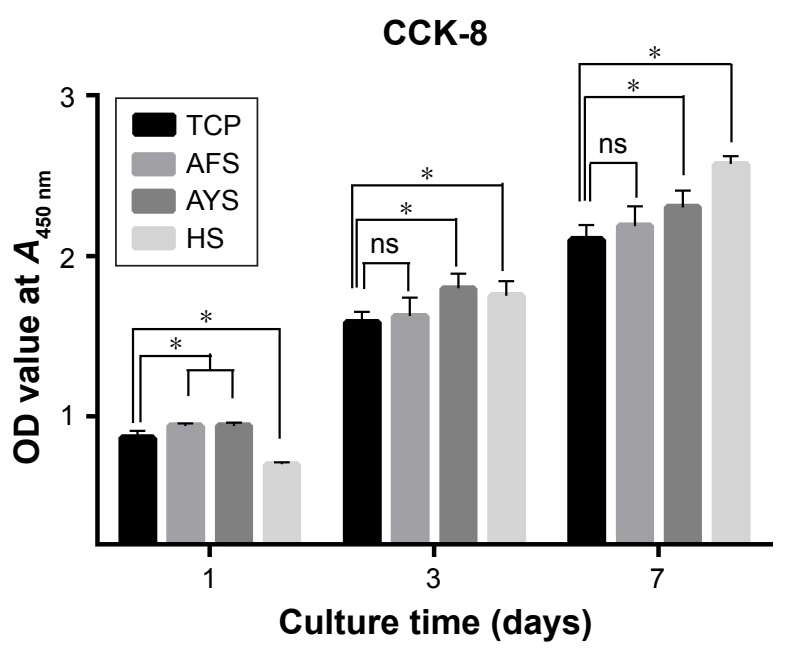

Figure 5 BMSCs' proliferation on different scaffolds by CCK-8 assay.

Notes: Results of BMSCs cultured on the AFS, AYS, and HS for up to 7 days. The data are expressed as the mean $\pm S D$. $* P<0.05$, a statistically significant difference between groups; ns, $P>0.05$, no significant difference between the two groups $(n=3)$.

Abbreviations: AFS, aligned nanofiber scaffolds; AYS, aligned nanoyarn scaffolds; BMSCs, bone marrow-derived mesenchymal stem cells; CCK-8, cell counting kit-8; HS, hybrid scaffold; TCP, tissue culture plate; SD, standard deviation. into AYS at a depth of $>150 \mu \mathrm{m}$, while the depth in AFS was $<50 \mu \mathrm{m}$, even though no significant difference existed in these two groups in terms of the median infiltration depth $(P>0.05)$. At this point of time, cells colonized on the boundary, with limited infiltration into the inner part of scaffold in AFS.

\section{Gene expression in BMSCs seeded on scaffolds}

Quantitative real-time PCR results showed that the mRNA expression level of COL1A1, a key phenotypical molecule in outer layer AF, was higher in AYS than in AFS or HS after culturing for 3 weeks in $\mathrm{CM}(P<0.05)$. No significant difference was observed between AFS and HS $(P>0.05)$ (Figure 10A). SOX-9, COL2A1, and Aggrecan mRNA expressions, which are inner AF markers, were upregulated in HS compared to AFS or AYS $(P<0.05)$ (Figure 10B-D). The "differentiation index", which is referred to as the ratio 

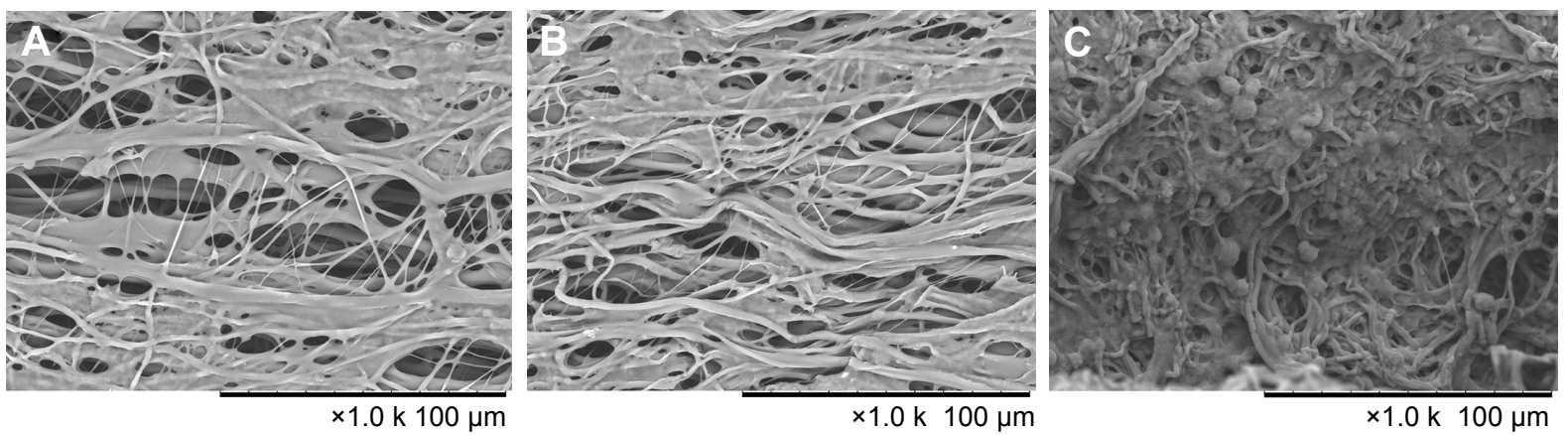

Figure 6 SEM images of BMSCs on scaffolds.

Notes: At day 7 after culture, BMSCs on AFS (A), AYS (B), and HS (C) were imaged. The scale bars in (A-C) represent $100 \mu \mathrm{m}$.

Abbreviations: AFS, aligned nanofiber scaffolds; AYS, aligned nanoyarn scaffolds; BMSCs, bone marrow-derived mesenchymal stem cells; HS, hybrid scaffold; SEM, scanning electron microscope.

of COL2A1 to COL1A1, was used to demonstrate the propensity for chondrogenesis. As shown in Figure 10E, the differentiation index in HS was significantly higher (fourfold) when compared with the other two groups $(P<0.05)$. No significant difference was observed between AFS and AYS $(P>0.05)$.

\section{Discussion}

Effective biological AF repair is an urgent clinical demand. Despite widespread investigation and considerable progress, products available for clinical applications in AF tissue engineering are scarce. In an effort to develop a biological tissue engineering scaffold for AF repair, we created a nanoyarn nanofibrous scaffold and a three-dimensional porous nanofiber/nanoyarn HS that partially mirrored several important functional signatures of the native AF. These constructs 1) had tensile and compressive mechanical properties similar to that of AF, 2) allowed cells to infiltrate between nanoyarns and three-dimensional porous structure, and 3) allowed differentiation of BMSCs into AF cells into their topographical framework in chemically defined conditions.

Successful tissue engineering of complex load-bearing tissues such as AF requires recapitulation of their topographical structure and mechanical functions. In contrast
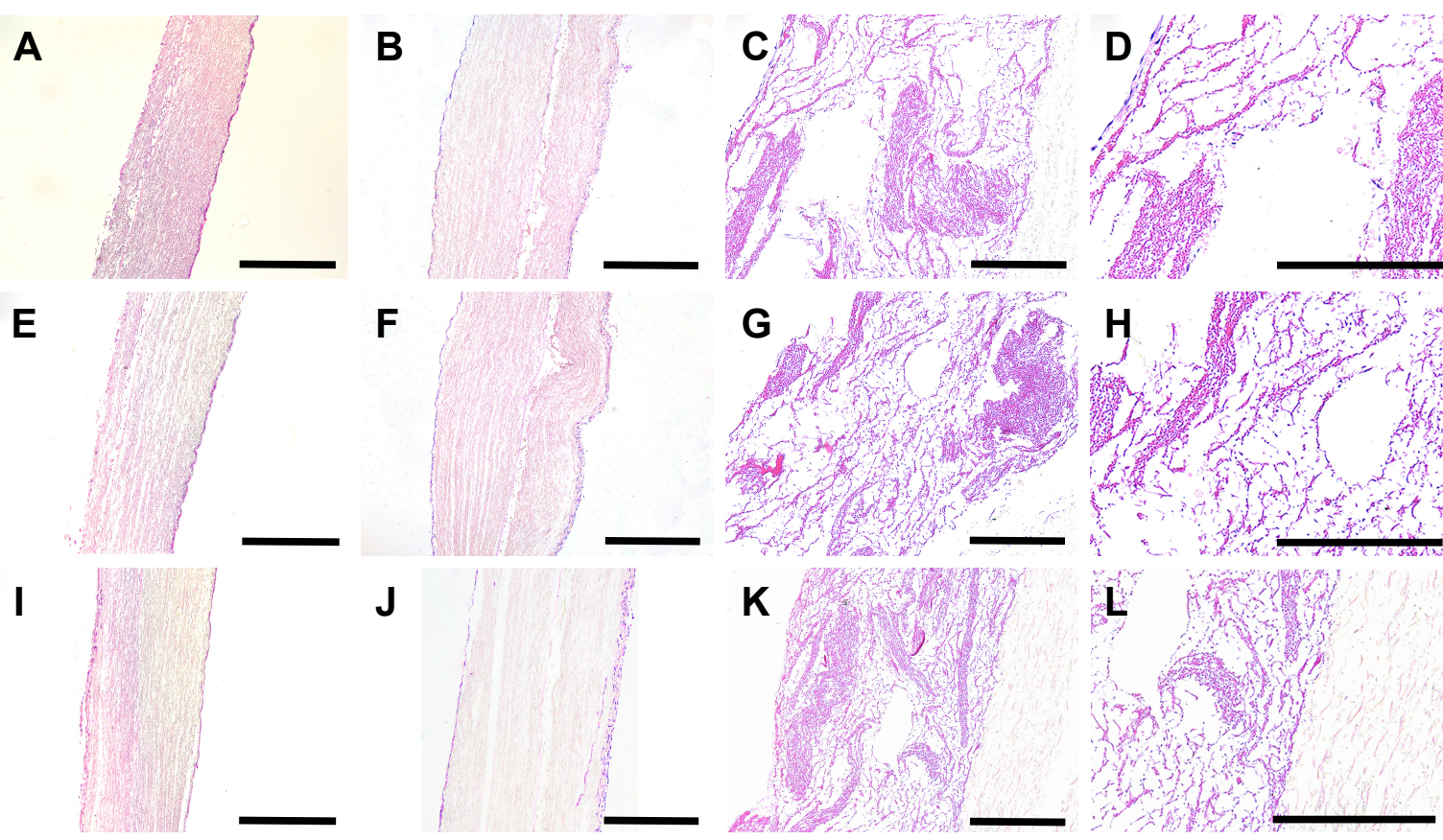

Figure 7 HE-stained histology images show BMSCs' distribution on different scaffolds up to 14 days.

Notes: AFS (A, E, and I), AYS (B, F, and J), and HS (C, D, G, H, K, L) at days 3 (A-D), 7 (E-H), and I4 (I-L), respectively. Panels (D, H, and L) were local amplification images of (C, $\mathbf{G}$, and $\mathbf{K})$. Scale bars: $200 \mu \mathrm{m}$ for all images.

Abbreviations: AFS, aligned nanofiber scaffolds; AYS, aligned nanoyarn scaffolds; BMSCs, bone marrow-derived mesenchymal stem cells; HE, hematoxylin and eosin; HS, hybrid scaffold. 

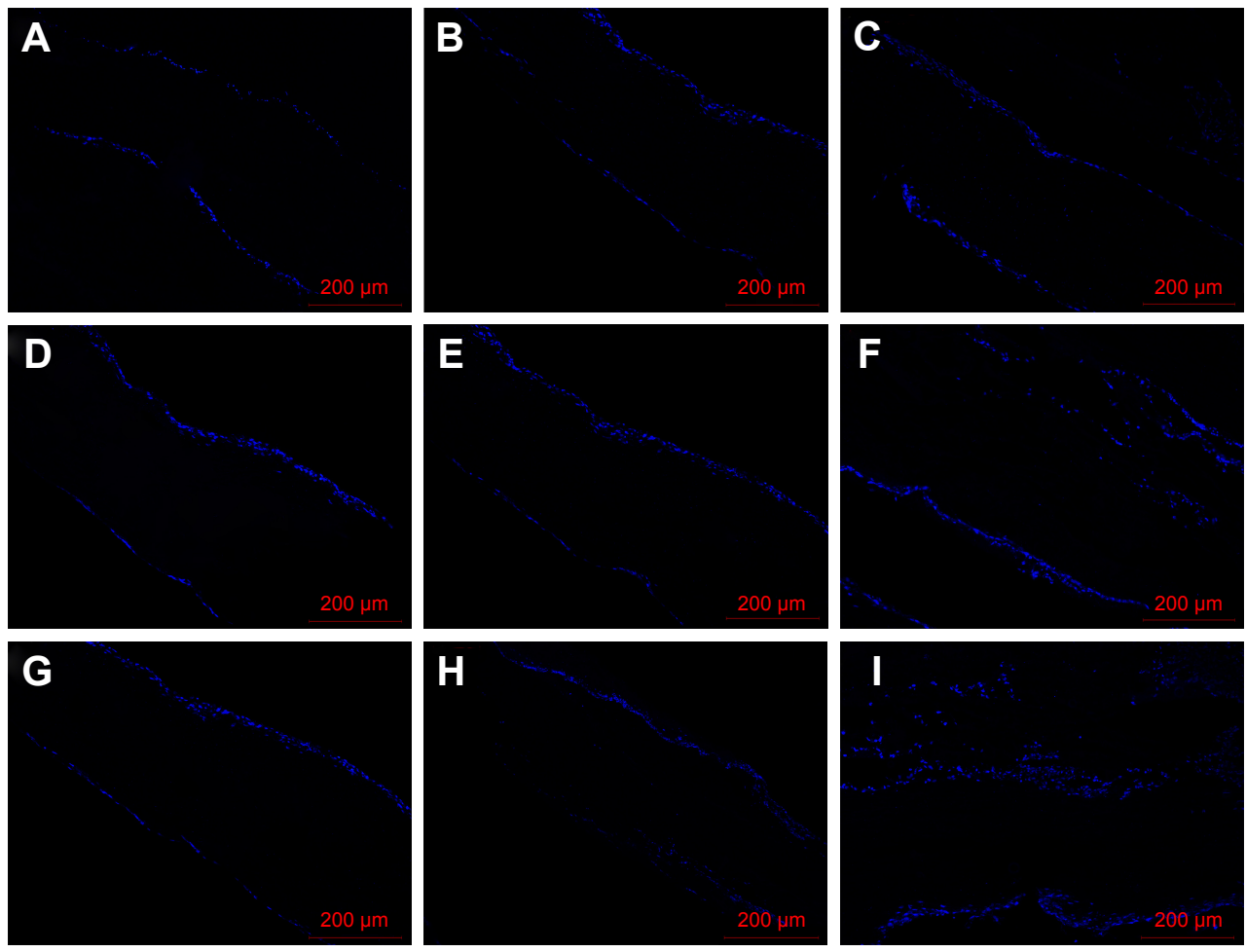

Figure 8 Infiltration of BMSCs into different scaffolds by DAPI.

Notes: BMSCs were seeded on different scaffolds up to 14 days, and cell nuclei were stained with DAPI. AFS (A, D, and G), AYS (B, E, and H), and HS (C, F, and I) at days 3 (A-C), 7 (D-F), and I4 (G-I). Scale bars: $200 \mu \mathrm{m}$ for all images.

Abbreviations: AFS, aligned nanofiber scaffolds; AYS, aligned nanoyarn scaffolds; BMSCs, bone marrow-derived mesenchymal stem cells; DAPI, 4',6-diamidino-2phenylindole; HE, hematoxylin and eosin; HS, hybrid scaffold.

to scaffolds previously used for AF tissue engineering, which included hydrogels ${ }^{13,31}$ and macroporous synthetic scaffolds, ${ }^{32,33}$ electrospinning permits the formation of arrays of aligned nanofibers to serve as a template for the deposition of a unidirectionally organized extracellular matrix by resident cells..$^{20}$ As demonstrated in our study, AYS, which is composed of nanoyarns connected and twisted by many nanofibers, has a larger fiber pore size and higher porosity compared to AFS. The porosity and correct pore size are fundamental characteristics that enable scaffolds to provide

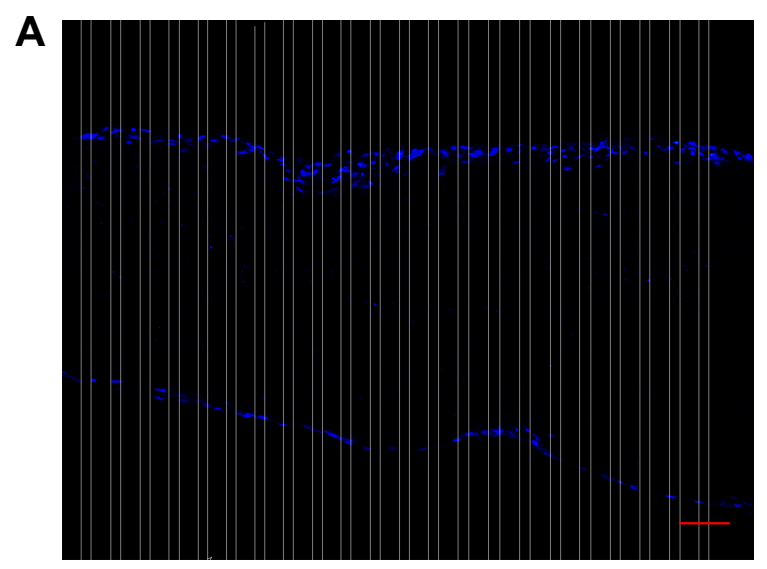

B

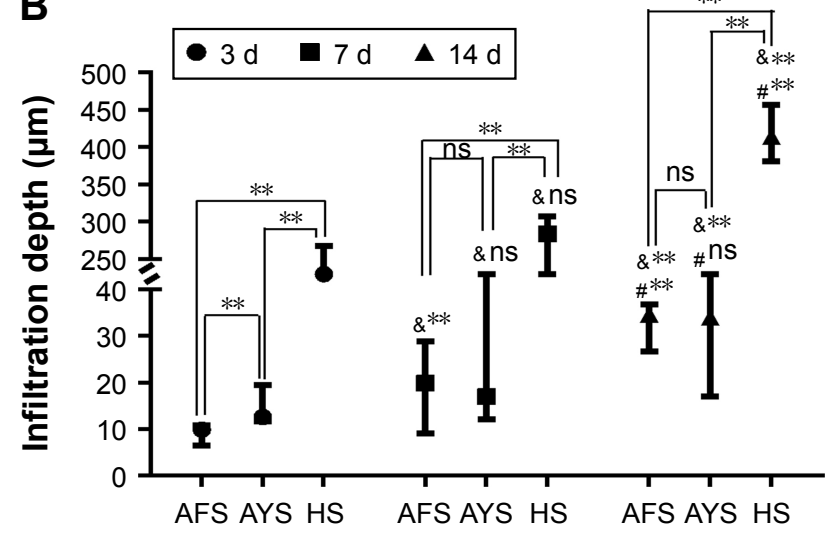

Figure 9 The infiltration depth of BMSCs in different scaffolds.

Notes: Infiltration depth was performed by overlaying an array of columns as depicted in (A) on the DAPI-stained section images of the different scaffolds. The deepest cell localization in each column was recorded and averaged for every sample group. The result is shown in (B). When compared with AFS, significant differences were observed in the infiltration depth of AYS and HS at each time point. The bar in (A) represents $50 \mu \mathrm{m}$. $* * P<0.0$ I, a statistically significant difference between groups; ns, no significant difference between the two groups; ${ }^{\star}$ Comparison of infiltration depth between day 7, 14 and day 3; \#Comparison of infiltration depth between day 7 and day 14 .

Abbreviations: AFS, aligned nanofiber scaffolds; AYS, aligned nanoyarn scaffolds; BMSCs, bone marrow-derived mesenchymal stem cells; DAPI, 4',6-diamidino-2phenylindole; HS, hybrid scaffold. 

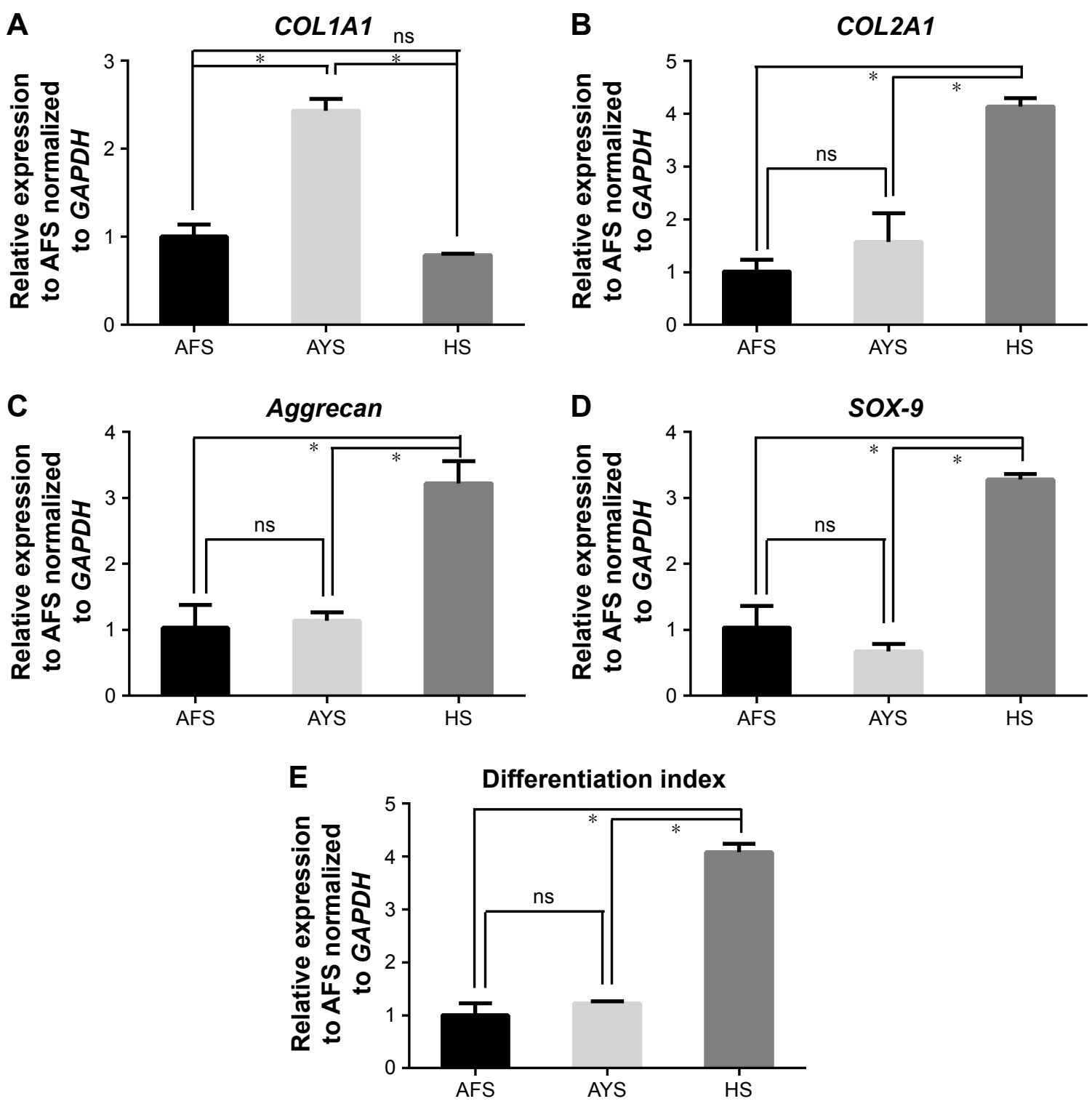

Figure 10 The quantitative real-time PCR was used to examine the mRNA expression of COLIAI (A), COL2AI (B), Aggrecan (C), SOX-9 (D), and "differentiation index" (E) of BMSCs on different scaffolds with CM for 3 weeks.

Notes: The differentiation index is the ratio of type II to type I collagens. Data are expressed as mean \pm SD (each group, $n=3$ ). * $* 0.05$, significant difference existed when compared between group; ns, $P>0.05$, no significant difference existed between group.

Abbreviations: AFS, aligned nanofiber scaffolds; AYS, aligned nanoyarn scaffolds; BMSCs, bone marrow-derived mesenchymal stem cells; CM, chondrogenic medium; HS, hybrid scaffold; SD, standard deviation.

space for cell adhesion and migration to allow nutrition diffusion and metabolite removal. ${ }^{34,35}$ AYS provided a relatively better three-dimensional and aligned microstructure that morphologically mimics the native AF tissue. Additionally, the nanoyarn scaffold exhibited a highly aligned structure, which was better than AFS.

AF exhibits spatial variations in structure and composition that gives rise to both anisotropy and heterogeneity in its material behavior in tension. ${ }^{14}$ Pilot experiments show that $\mathrm{AF}$ tissue has unique mechanical characteristics such as viscoelasticity, anisotropy, and a nonlinear behavior. ${ }^{14}$
The circumferential tensile modulus of outer layer AF may reach up to $17.4 \pm 14.3 \mathrm{MPa} .{ }^{14}$ In our study, the tensile modulus of AFS and AYS in the parallel direction achieved $13.46 \pm 0.54$ and $10.39 \pm 0.46 \mathrm{MPa}$, respectively, and this partially approximates the circumferential tensile modulus of the outer layer of human AF. The tensile modulus of the scaffold could potentially be increased by combining the scaffolds with BMSCs to promote extracellular matrix accumulation and remodeling by achieving proper porosity and pore size to match the tensile strength of AF. Baker et $a l^{36}$ developed an enabling technology in which tunable composite nanofibrous 
scaffolds were produced to enable functional tissue formation by adding water-soluble sacrificial poly(ethylene oxide). The removal of sacrificial poly(ethylene oxide) fibers can enhance scaffold porosity and cell infiltration, which in turn may lead to pronounced increase in the tensile properties of the engineered constructs.

The three scaffolds in our study did not have any inhibitory effects on BMSCs' proliferation in vitro, indicating good biocompatibility. These results also indicate that AYS, and especially HS, can provide a better structure and space for cell proliferation than AFS and TCP owing to their larger pore size and higher porosity. BMSCs spread along the fibers in aligned nanofiber and nanoyarn scaffolds and exhibited a spindle-shaped cellular morphology, while adapting a more rounded shape with assembled blocks in the hybrid porous scaffolds. The diverse cell morphology suggests that the BMSCs may be able to sense the topography of scaffold substrates and transform into different morphological assemblies. This is consistent with the findings that cellular behavior including proliferation, differentiation, and migration is influenced by nanofiber topographic structure, such as pore size, fiber diameter, and fiber alignment. ${ }^{37}$

While aligned nanofiber scaffolds are promising candidates for AF regeneration, several limitations remain. Most significantly, cell infiltration into these scaffolds is slow because of dense fiber packing, reduced pore size, and limited cellular ingress. To address this problem, a number of methods for improving infiltration have been proposed, such as selective removal of sacrificial fibers, ${ }^{38}$ salt leaching, ${ }^{39}$ cryogenic electrospinning, ${ }^{40}$ and introducing nanoyarn fiber. ${ }^{24}$ Conjugated electrospinning, a simple and versatile technique to manufacture aligned nanofibers and nanoyarns, is constructed by two simultaneous opposite voltage sprays. ${ }^{41}$ Unlike the conventional technique of using a rotating drum to collect aligned nanofibers, the fibers developed by conjugated electrospinning attract each other, stick together, and form neutral yarns with a high degree of alignment. As shown in our study, AYS had a higher extent of alignment and a relatively larger porosity and pore size compared to AFS, which may provide a three-dimensional environment for cell-scaffold interaction and accelerate cell infiltration into the scaffold. Notably, parts of BMSCs had ingrown into AYS at a depth of $>150 \mu \mathrm{m}$ after in vitro culture for 14 days, while the depth in AFS was $<50 \mu \mathrm{m}$, though no significant difference was observed in terms of the median infiltration depth. Electrospun nanoyarn scaffolds constructed by dynamic liquid supporting system demonstrated higher porosity and excellent cell infiltration. However, the maximal tensile strength of these scaffolds is $\sim 4 \mathrm{MPa}$, which is a big gap to be used for AF tissue engineering. ${ }^{28}$ In general, the mechanical properties of a scaffold decrease with increasing porosity. Therefore, it is necessary to search for a trade-off between these two opposite parameters in order to meet the requirements of scaffolds for $\mathrm{AF}$ tissue engineering in a future study.

Given the intimate relationship between function and mechanical parameters, biomimetic scaffolds that replicate essential anatomic features of the tissue of interest are of great importance for tissue engineering application. Although many studies have matched AF tissue with tensile properties, due to the complexity of the IVD, there is no consensus on what additional mechanical properties must be attained and to what extent. Because AF must simultaneously withstand stress due to axial loading of the spine as well as torsion, it is possible that the AF mimetic engineered scaffold with anticompression function should be structurally optimized to reach functional equivalence of their native counterparts. In order to partially mimic the axial compression property of AF and increase cell infiltration rate further, we constructed the HS with AYS and 3-DPS by freeze-drying method and cross-linking. Our previous work verified the maximum compressive stress of 3-DPS to be $\sim 0.35 \mathrm{MPa}$, which is nearly half of the native human AF tissues in the axial compressive modulus $(0.8 \pm 0.9 \mathrm{MPa}){ }^{14}$ 3-DPS was assembled by an open-cell cellular architecture and formed an elastic interconnected network with good reversible super-elasticity. Moreover, 3-DPS was assembled by nanofibers with hierarchical cellular structure, which may be favorable for cell adhesion, proliferation, and infiltration. Different sizes of pores, from hundreds to thousand micrometer square, allow cell ingress even during early period of cell culture $(-236.43 \mu \mathrm{m}$ after 3 days culture), whereas limited cell infiltration on the periphery of the scaffold was observed in AFS. After 14 days of culture, BMSCs infiltrated to a depth of $\sim 500 \mu \mathrm{m}$ in HS. Parts of BMSCs had ingrown into AYS at a depth of $>150 \mu \mathrm{m}$, while the deepest in AFS was $<50 \mu \mathrm{m}$. This may contributed to the increased porosity with the inclusion of larger pores in 3-DPS and nanoyarns in AYS. In this respect, HS appears to be the best candidate among these three types of scaffolds in terms of mechanical property and cell infiltration and may be suitable for clinical application for the treatment of damaged AF tissue in vivo.

Comparable topographical geometry and mechanical features between the chosen scaffold and the native tissue allow cells to retain their characteristic cellular morphology, which has been shown to play an essential role in maintaining 
the cellular phenotype. As specific phenotypes to distinguish AF cells are limited and there is no consensus regarding the differentiation of BMSCs to AF cell, ${ }^{42,43}$ we only analyzed the chondrogenic markers to evaluate BMSCs' differentiation toward AF cells in this present study similar to a previous report. ${ }^{44}$ Our results indicated that $C O L 1 A 1$ expression level was higher in AYS, while SOX-9, COL2A1, and Aggrecan expression levels were higher in HS compared to other scaffolds. The differentiation index, defined by the ratio of gene expression of type II to type I collagen, is a known method to track the functionality of chondrocytes or chondrocyte-like cells in case of disk cells ${ }^{45,46}$ and may be used as a reference to measure chondrogenesis differentiation tendency of BMSCs into disk cells. The differentiation index in HS was significantly increased by fourfold when compared with other two groups. Recent study showed that physical cues from aligned or randomly oriented nanofiber scaffold would influence the cellular behavior of BMSCs and would induced lineage commitment depending on the cytoskeleton-mediated mechanotransduction. ${ }^{47}$ The increased expression of COL1A1 in AYS may be caused by topography-induced BMSCs' differentiation into fibrous cartilage-like tissue, similar to outer layer AF tissue. An outstanding work conducted by Nerurkar et al, ${ }^{21}$ which used nanofibrous scaffolds seeded with BMSCs in specific chondrogenic differentiation medium, demonstrated that these scaffolds directed the deposition of organized, collagen-rich extracellular matrix that mimicked the multilamellar architecture of AF. The porous structure in HS may provide optimal conditions for cell attachment and assembly and further affect BMSCs' chondrogenesis differentiation and formation of extracellular matrix similar to the inner AF tissue.

Although the present work promises further advancement in AF engineering, it also highlights several challenges that must be addressed before these scaffolds could be used in vivo. The first issue is the low cell infiltration efficiency in AFS and AYS with a large proportion of BMSCs distributed on the surface of these scaffolds, even though parts of BMSCs had ingressed in the inner portion of AYS. The nonuniform distribution may have been the result of dense fibers' packing in AFS and a static culture after seeding. A previous study has also implied the limited infiltration of cells in AFS in a static state. ${ }^{48}$ These limitations might be overcome by using dynamic culture or perfusion culture conditions in vitro. In addition, further work needs to be done to optimize the conditions of BMSCs' differentiation toward AF cells. As the current technology moves toward repairing AF defect in vivo, it will be critically important to develop suitable implantation and fixation technique for integration into the surrounding tissue, as well as the physiological constraints necessary to sustain its location. It remains to be determined whether this will occur under in vivo loading conditions due to the complexity of the IVD. Hopefully, with the development of minimally invasive spine surgery instruments, it would be feasibly accessible to implant the scaffold and immobilize it to allow integration during the surgical procedure.

\section{Conclusion}

This study has developed a HS composed of two distinct topographies, a nanoyarn layer and a porous nanofiber layer, that replicates the tensile strength, axial compression, and anisotropic property of AF tissue to some degree. Biocompatibility analyses demonstrated that AYS and HS yield improved cell proliferation. Moreover, HS provides a novel three-dimensional platform for BMSCs' proliferation, infiltration, and differentiation toward AF cells. This study demonstrates that the HS could balance the mechanical property and BMSCs' infiltration and may provide a promising approach to the generation of equivalent replacement scaffold for AF repair.

\section{Acknowledgment}

This study was financially supported by the National Natural Science Foundation of China (grant NO 81472071 and 81772445).

\section{Disclosure}

The authors report no conflicts of interest in this work.

\section{References}

1. Ruttimann B. [A historical approach to herniated disk]. Schweiz Rundsch Med Prax. 1990;79(25):791-796.

2. Yorimitsu E, Chiba K, Toyama Y, Hirabayashi K. Long-term outcomes of standard discectomy for lumbar disc herniation: a follow-up study of more than 10 years. Spine (Phila Pa 1976). 2001;26(6):652-657.

3. Yeung AT, Tsou PM. Posterolateral endoscopic excision for lumbar disc herniation: surgical technique, outcome, and complications in 307 consecutive cases. Spine (Phila Pa 1976). 2002;27(7):722-731.

4. Atlas SJ, Keller RB, Wu YA, et al. Long-term outcomes of surgical and nonsurgical management of sciatica secondary to a lumbar disc herniation: 10 year results from the maine lumbar spine study. Spine (Phila Pa 1976). 2005;30(8):927-935.

5. Peul WC, van den Hout WB, Brand R, Thomeer RT, Koes BW; LeidenThe Hague Spine Intervention Prognostic Study Group. Prolonged conservative care versus early surgery in patients with sciatica caused by lumbar disc herniation: two year results of a randomised controlled trial. BMJ. 2008;336(7657):1355-1358.

6. Carragee EJ, Han MY, Suen PW, Kim D. Clinical outcomes after lumbar discectomy for sciatica: the effects of fragment type and anular competence. J Bone Joint Surg Am. 2003;85-A(1):102-108.

7. McGirt MJ, Eustacchio S, Varga P, et al. A prospective cohort study of close interval computed tomography and magnetic resonance imaging after primary lumbar discectomy: factors associated with recurrent disc herniation and disc height loss. Spine (Phila Pa 1976). 2009;34(19):2044-2051. 
8. Ahlgren BD, Lui W, Herkowitz HN, Panjabi MM, Guiboux JP. Effect of anular repair on the healing strength of the intervertebral disc: a sheep model. Spine (Phila Pa 1976). 2000;25(17):2165-2170.

9. Bailey A, Araghi A, Blumenthal S, Huffmon GV; Anular Repair Clinical Study Group. Prospective, multicenter, randomized, controlled study of anular repair in lumbar discectomy: two-year follow-up. Spine (Phila Pa 1976). 2013;38(14):1161-1169.

10. Lequin MB, Barth M, Thome C, Bouma GJ. Primary limited lumbar discectomy with an annulus closure device: one-year clinical and radiographic results from a prospective, multi-center study. Korean J Spine. 2012;9(4):340-347.

11. Wilke HJ, Ressel L, Heuer F, Graf N, Rath S. Can prevention of a reherniation be investigated? Establishment of a herniation model and experiments with an anular closure device. Spine (Phila Pa 1976). 2013; 38(10):E587-E593.

12. Parker SL, Grahovac G, Vukas D, et al. Effect of an annular closure device (Barricaid) on same level recurrent disc herniation and disc height loss after primary lumbar discectomy: two-year results of a multi-center prospective cohort study. Clin Spine Surg. 2016;29(10):454-460.

13. Likhitpanichkul M, Dreischarf M, Illien-Junger S, et al. Fibrin-genipin adhesive hydrogel for annulus fibrosus repair: performance evaluation with large animal organ culture, in situ biomechanics, and in vivo degradation tests. Eur Cell Mater. 2014;28:25-37; discussion 37-38.

14. Elliott DM, Setton LA. Anisotropic and inhomogeneous tensile behavior of the human anulus fibrosus: experimental measurement and material model predictions. J Biomech Eng. 2001;123(3):256-263.

15. Mauck RL, Baker BM, Nerurkar NL, et al. Engineering on the straight and narrow: the mechanics of nanofibrous assemblies for fiber-reinforced tissue regeneration. Tissue Eng Part B Rev. 2009;15(2):171-193.

16. Nerurkar NL, Elliott DM, Mauck RL. Mechanics of oriented electrospun nanofibrous scaffolds for annulus fibrosus tissue engineering. J Orthop Res. 2007;25(8):1018-1028.

17. Yang L, Kandel RA, Chang G, Santerre JP. Polar surface chemistry of nanofibrous polyurethane scaffold affects annulus fibrosus cell attachment and early matrix accumulation. J Biomed Mater Res A. 2009;91(4): 1089-1099.

18. Gruber HE, Hoelscher G, Ingram JA, Hanley EN Jr. Culture of human anulus fibrosus cells on polyamide nanofibers extracellular matrix production. Spine. 2009;34(1):4-9.

19. Nesti LJ, Li WJ, Shanti RM, et al. Intervertebral disc tissue engineering using a novel hyaluronic acid-nanofibrous scaffold (HANFS) amalgam. Tissue Eng Part A. 2008;14(9):1527-1537.

20. Nerurkar NL, Mauck RL, Elliott DM. ISSLS prize winner: integrating theoretical and experimental methods for functional tissue engineering of the annulus fibrosus. Spine (Phila Pa 1976). 2008;33(25): 2691-2701.

21. Nerurkar NL, Baker BM, Sen S, Wible EE, Elliott DM, Mauck RL. Nanofibrous biologic laminates replicate the form and function of the annulus fibrosus. Nat Mater. 2009;8(12):986-992.

22. Nerurkar NL, Sen S, Huang AH, Elliott DM, Mauck RL. Engineered disc-like angle-ply structures for intervertebral disc replacement. Spine. 2010;35(8):867-873.

23. Chen W, Ma J, Zhu L, et al. Superelastic, superabsorbent and 3D nanofiber-assembled scaffold for tissue engineering. Colloids Surf B Biointerfaces. 2016;142:165-172.

24. Si Y, Yu J, Tang X, Ge J, Ding B. Ultralight nanofibre-assembled cellular aerogels with superelasticity and multifunctionality. Nat Commun. 2014;5:5802.

25. Gough JE, Scotchford CA, Downes S. Cytotoxicity of glutaraldehyde crosslinked collagen/poly(vinyl alcohol) films is by the mechanism of apoptosis. J Biomed Mater Res. 2002;61(1):121-130.

26. Marchand $\mathrm{F}$, Ahmed AM. Investigation of the laminate structure of lumbar disc anulus fibrosus. Spine (Phila Pa 1976). 1990;15(5): 402-410.

27. Yang C, Deng G, Chen W, Ye X, Mo X. A novel electrospun-aligned nanoyarn-reinforced nanofibrous scaffold for tendon tissue engineering. Colloids Surf B Biointerfaces. 2014;122:270-276.
28. Xu Y, Wu J, Wang H, et al. Fabrication of electrospun poly(L-lactideco-epsilon-caprolactone)/collagen nanoyarn network as a novel, three-dimensional, macroporous, aligned scaffold for tendon tissue engineering. Tissue Eng Part C Methods. 2013;19(12):925-936.

29. Reilly GC, Radin S, Chen AT, Ducheyne P. Differential alkaline phosphatase responses of rat and human bone marrow derived mesenchymal stem cells to 45S5 bioactive glass. Biomaterials. 2007;28(28): 4091-4097.

30. Pham QP, Sharma U, Mikos AG. Electrospun poly(epsilon-caprolactone) microfiber and multilayer nanofiber/microfiber scaffolds: characterization of scaffolds and measurement of cellular infiltration. Biomacromolecules. 2006;7(10):2796-2805.

31. Thonar E, An H, Masuda K. Compartmentalization of the matrix formed by nucleus pulposus and annulus fibrosus cells in alginate gel. Biochem Soc Trans. 2002;30(pt 6):874-878.

32. Wan Y, Feng G, Shen FH, Balian G, Laurencin CT, Li X. Novel biodegradable poly(1,8-octanediol malate) for annulus fibrosus regeneration. Macromol Biosci. 2007;7(11):1217-1224.

33. Pirvu T, Blanquer SB, Benneker LM, et al. A combined biomaterial and cellular approach for annulus fibrosus rupture repair. Biomaterials. 2015;42:11-19.

34. Agrawal CM, Ray RB. Biodegradable polymeric scaffolds for musculoskeletal tissue engineering. J Biomed Mater Res. 2001;55(2):141-150.

35. Zeltinger J, Sherwood JK, Graham DA, Müeller R, Griffith LG. Effect of pore size and void fraction on cellular adhesion, proliferation, and matrix deposition. Tissue Eng. 2001;7(5):557-572.

36. Baker BM, Shah RP, Silverstein AM, Esterhai JL, Burdick JA, Mauck RL. Sacrificial nanofibrous composites provide instruction without impediment and enable functional tissue formation. Proc Natl Acad Sci U S A. 2012;109(35):14176-14181.

37. Beachley V, Wen X. Polymer nanofibrous structures: fabrication, biofunctionalization, and cell interactions. Prog Polym Sci. 2010;35(7): 868-892.

38. Martin JT, Milby AH, Chiaro JA, et al. Translation of an engineered nanofibrous disc-like angle-ply structure for intervertebral disc replacement in a small animal model. Acta Biomater. 2014;10(6):2473-2481.

39. Nam J, Huang Y, Agarwal S, Lannutti J. Improved cellular infiltration in electrospun fiber via engineered porosity. Tissue Eng. 2007;13(9): 2249-2257.

40. Leong MF, Rasheed MZ, Lim TC, Chian KS. In vitro cell infiltration and in vivo cell infiltration and vascularization in a fibrous, highly porous poly(D,L-lactide) scaffold fabricated by cryogenic electrospinning technique. J Biomed Mater Res A. 2009;91(1):231-240.

41. Pan H, Li L, Hu L, Cui X. Continuous aligned polymer fibers produced by a modified electrospinning method. Polymer. 2006;47(14):4901-4904.

42. Evans C. Potential biologic therapies for the intervertebral disc. J Bone Joint Surg Am. 2006;88(suppl 2):95-98.

43. Pattappa G, Li Z, Peroglio M, Wismer N, Alini M, Grad S. Diversity of intervertebral disc cells: phenotype and function. J Anat. 2012;221(6): 480-496.

44. Tsai TL, Nelson BC, Anderson PA, Zdeblick TA, Li WJ. Intervertebral disc and stem cells cocultured in biomimetic extracellular matrix stimulated by cyclic compression in perfusion bioreactor. Spine J. 2014; 14(9):2127-2140.

45. Yang KG, Saris DB, Verbout AJ, Creemers LB, Dhert WJ. The effect of synovial fluid from injured knee joints on in vitro chondrogenesis. Tissue Eng. 2006;12(10):2957-2964.

46. Zhang H, Wang L, Park JB, et al. Intradiscal injection of simvastatin retards progression of intervertebral disc degeneration induced by stab injury. Arthritis Res Ther. 2009;11(6):R172.

47. Yin Z, Chen X, Song HX, et al. Electrospun scaffolds for multiple tissues regeneration in vivo through topography dependent induction of lineage specific differentiation. Biomaterials. 2015;44:173-185.

48. Nerurkar NL, Sen S, Baker BM, Elliott DM, Mauck RL. Dynamic culture enhances stem cell infiltration and modulates extracellular matrix production on aligned electrospun nanofibrous scaffolds. Acta Biomater. 2011;7(2):485-491. 
International Journal of Nanomedicine

Dovepress

\section{Publish your work in this journal}

The International Journal of Nanomedicine is an international, peerreviewed journal focusing on the application of nanotechnology in diagnostics, therapeutics, and drug delivery systems throughout the biomedical field. This journal is indexed on PubMed Central, MedLine, CAS, SciSearch ${ }^{\circledR}$, Current Contents ${ }^{\circledR} /$ Clinical Medicine,
Journal Citation Reports/Science Edition, EMBase, Scopus and the Elsevier Bibliographic databases. The manuscript management system is completely online and includes a very quick and fair peer-review system, which is all easy to use. Visit http://www.dovepress.com/ testimonials.php to read real quotes from published authors.

Submit your manuscript here: http://www.dovepress.com/international-journal-of-nanomedicine-journal 